DOI 10.2478/aucft-2014-0016

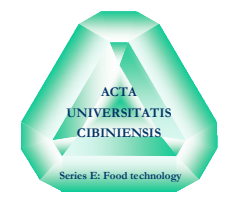

\title{
IDENTIFICATION OF PROBIOTIC STRAINS FROM HUMAN MILK IN BREASTFED INFANTS WITH RESPIRATORY INFECTIONS
}

\author{
Bogdan NEAMTU $*^{1}$, Ovidiu TITA***, Mihai NEAMTU*, Mihaela \\ TITA $^{* * *}$, Mirela HILA**, Ionela MANIU**** \\ * "Lucian Blaga" University of Sibiu, Faculty of Medicine "Victor Papilian", \\ str. Lucian Blaga, nr. 2A, Sibiu 550169, Romania \\ **Pediatric Clinical Hospital from Sibiu, Pompeiu Onofrei Street, no: 2-4, \\ 550166 Sibiu, Romania \\ ***"Lucian Blaga" University of Sibiu, Faculty of Agricultural Sciences, \\ Food Industry and Environment Protection, Romania \\ ****"Lucian Blaga" University of Sibiu, Faculty of Sciences, Department of \\ Mathematics and Informatics, Romania
}

\begin{abstract}
Isolation and industrial exploitation of probiotics from human milk is a goal for worldwide milk biotechnology centres because of their modulation effect on the immune system in infants and adults. In the proposed study we have analysed fermentation patterns of Lactobacilli isolated from human milk, the reliability of API $50 \mathrm{CH}$ carbohydrate fermentation system and a possible link between lactose concentrations and fermentation profiles on carbohydrates. We had succesfully identified three species of Lactobacillus (paracasei ssp paracasei, fermentum, acidophilus) and one unsatisfactory identification of Lactoccocus lactis ssp lactis. These strains had different carbohydrate fermentation patterns but with common characteristics and showed no statistically significant correlations between their carbohydrate metabolic trends and lactose concentrations in the milk samples.
\end{abstract}

Keywords: human milk, lactobacilli, API 50CH identification, lactose concentrat

\section{INTRODUCTION}

Probiotics are "living microorganisms which in adequate amounts bring

\footnotetext{
${ }^{1}$ Corresponding author. Adress: "Lucian Blaga" University of Sibiu, Faculty of Medicine "Victor Papilian", str. Lucian Blaga , nr. 2A, Sibiu 550169, Romania. E-mail: bogdanneamtu76@gmail.com
}

Vol. XVIII (2014), no. 2 
benefits for the host" (FAO/WHO, 2002). Their roles correlate with the type of strain (Burgain et al., 2011),(Agrawal 2005). Probiotics are represented by many microorganisms in the genus Lactobacillus prokaryotic bacteria, about 150 species so far (O'Donnell et al., 2013) and the genus Bifidobacterium about 30 species, but also in the field of fungi (some yeasts) (Burgain et al., 2011).

Isolation and industrial development of probiotics from human milk is a real challenge and a goal for milk microbiology and biotechnology centres from around the globe. Human milk is an important source of probiotic strains for infant intestinal microbiota development (Malek et al. 2010), (Yavuzdurmaz et al., 2011). Clinical studies have showed unequivocally a hospitalization rate three times higher in infants fed with milk powder formula compared with breastfed infants admitted with respiratory infections. It seems that microbiota from human milk modulates the immune response in infants(Chirico et al., 2008). Most probably these strains use lactose as a principal substrate for energy and growth since lactose concentrations are much higher compared with oligosaccharides in human milk. Nevertheless, genus Lactobacillus has broad metabolic capacity in many cases with specific traits for each strain(O'Donnell et al., 2013). Current research projects related to different species of Lactobacillus investigate the impact of milk milieu on the bacterial genome expression especially the promotion of probiotic characteristics and metabolic variability (Klaenhammer et al., 2014). More research data is needed in analysing this complex subject. Probiotic conventional phenotypic identification by carbohydrates fermentation method reflects in fact the proportion of the carbohydrate catabolic genes expressed by each type of strain under particular cultivation conditions (Leuschner et al., 2002). Sugars fermentation method is widely used to identify probiotics in isolates from various sources: 1 . Human milk (Malek et al., 2010),(Yavuzdurmaz et al., 2011); 2. Animal milk kefir (Zanisic et al., 2012),(Hyun jue Kim et al., 2006); 3. Dairy-yogurt (Karna et al., 2007), (Kizerwetter et al., 2005); 4. Fermented products like meat, dairy, vegetables, fruit, pastry, drinks; 5. Animal or human digestive tracts (Ashraf et al., 2009),(Zanisic et al., 2012),(Khalil et al., 2007); 6. Genital Tracts (Herbel et al., 2013).

In our study we have analysed fermentation patterns of lactobacilli species isolated from human milk and the reliability of API $50 \mathrm{CH}$ carbohydrate fermentation system as a first step identification method in the process of industrial exploitation of these strains. We have hypothesized that constant exposure to breast milieu, particularly human milk lactose levels could affect in a specific manner carbohydrate catabolic genes expression of these

Vol. XVIII (2014), no. 2 
lactic acid bacteria (metabolic capability).In this connection it was interesting to examine the possibility of a link between lactose concentrations and fermentation profiles on carbohydrates with a direct implication in the percentage of identification, $\mathrm{T}$ index and validation on a apiweb ${ }^{\mathrm{TM}}$ database.

\section{MATERIALS AND METHODS}

In the proposed study we have collected breast milk samples from the mothers admitted in our clinic whose infants were hospitalized for acute infections of respiratory pathways. Samples were harvested using sterile gloves with respect to local antisepsis (areola disinfected) following the methodology described by Yavuzdurmaz et al.(2011): 52 control samples from the skin without desinfection, 52 samples of breast milk from healthy mothers nursing infants with lower respiratory infections, in duplicates. Evaluation of human milk lactose levels was made using an ultrasonic analyzer. Milk samples were centrifuged at $3.000 \mathrm{rpm}$ for 20 minutes. Supernatant represented by the fatty substance was separated and the levels of lactose were determined on ultrasonic analyzer Ekomilk®. Samples from each milk probe were added in the specific medium for Lactobacilli MRS (Man Rogosa Sharpe) using streak plate technique. Isolates were examined according to the colony morphology, catalase reaction, gram staining and sugar fermentation tests (Leuschner et al., 2002). Isolates from MRS agar were passed then on MRS broth and kept in culture with glycerol $40 \%(\mathrm{v} / \mathrm{v})$ at $-80{ }^{\circ} \mathrm{C}$ in freezer. We have selected from 52 strains, 10 strains who have survived to multiple freeze-thaw cycles. These strains were evaluated as potential candidates for industrial exploitation because of their resistance.

Bacterial identification was carried out by means of galleries API $50 \mathrm{CH}$ (BioMerieux, France), a standardized system which combines 50 biochemical assays for the study of the microorganisms carbohydrate metabolic profile. API $50 \mathrm{CH}$ galleries were used in conjunction with the API 50 CHL medium to identify the genus and the species of lactobacilli. The prepared inoculum(density of the bacterial suspension 2 Mc Farland) was incubated in each tube of the 50 galleries at $37^{\circ} \mathrm{C}$ for 48 hours for each strain (10 tests of 50 tubes for each of the 10 strains). After 48 hours of incubation, acid production in the tubes turned color from blue/purple to yellow. In the tube containing the substrate esculin the color turned black. The summary results were noted for each tube in part: positive tubes at which the color turned yellow, meaning that the strain ferments sugars. A color with shade between green and yellow was considered as unsatisfactory (Kos

Vol. XVIII (2014), no. 2 
et al., 2008). Negative reactions were assigned for those tubes that have kept blue, meaning that in the respective probes the strain didn't ferment sugars. Bacterial identification was performed using the dedicated software apiweb version 5.1 (Biomerieux, France) which compared biochemical profile obtained for each strain with existing data.

We have evaluated for each strain the percentage of profile's identification relative to other similar taxa in the database. $\mathrm{T}$ index estimated how close was the profile from the typical reaction set for each taxon listed in the API 50 CHL Analytical Profile Index (number of ranges between 0 and 1) and was inversely proportional to the number of atypical tests. An excellent identification required a rate of over $99 \%$ and $\mathrm{T}$ index more than 0.75 . Computer cluster analysis of the fermentation profiles was performed based on the calculation of Pearson corelation's coefficient and average linkage analysis using Apriori algorithm on SPPS modeler version 14.2 (IBM). Pearson's correlations between lactose concentration, identification rate and $\mathrm{T}$ index were realised on SPSS version 19 (IBM).

\section{RESULTS AND DISCUSSIONS}

In our study we have identified 10 strains of lactic acid bacteria to survive to multiple freeze-thaw cycles, namely: 7 strains of Lactobacillus paracasei ssp paracasei, 1 strain of Lactobacillus fermentum , 1 strain of Lactobacillus acidophilus, one strain of Lactococcus lactis ssp lactis (Figure 1).

All the isolates belonging to Lactobacillus paracasei ssp paracasei strains (L6, L8, L15, L16, L18, L21, L22) were able to ferment ribose, galactose, glucose, fructose, mannose, lactose, mannitol, sorbitol, n-acetylglucosamine, amygdaline, arbutine, esculine, salicine, cellobiose, maltose, saccharose, trehalose, melezitose and gluconate, showing some differences with the findings published by Kadere et al., (2012) regarding inuline, turanose, tagatose, sorbose (Figure 1).

Lactobacillus fermentum strain (L1) fermented arabinose, ribose, raffinose, galactose, glucose, fructose, mannose, lactose, melibiose, esculine, salicine, maltose, saccharose. Maroki et al., (2011) described for this species also the ability of fermenting rhamnose and tagatose. Lactobacillus acidophilus strain (L3) has shown a carbohydrate utilisation pattern including salicine, n-acetyl-glucosamine, inuline, saccharose, raffinose, mannose, galactose, glucose, fructose, lactose, arbutine, cellobiose, maltose. Unfortunately available data from other studies suggest that the use of the API $50 \mathrm{CH}$ database for identification of commensal Lactobacillus species could lead to misidentification or

Vol. XVIII (2014), no. 2 
uninterpretable results. Boyd et al( 2005) have found that over half of the Lactobacillus jensenii and Lactobacillus gasseri isolates were erroneously identified as Lactobacillus acidophilus.

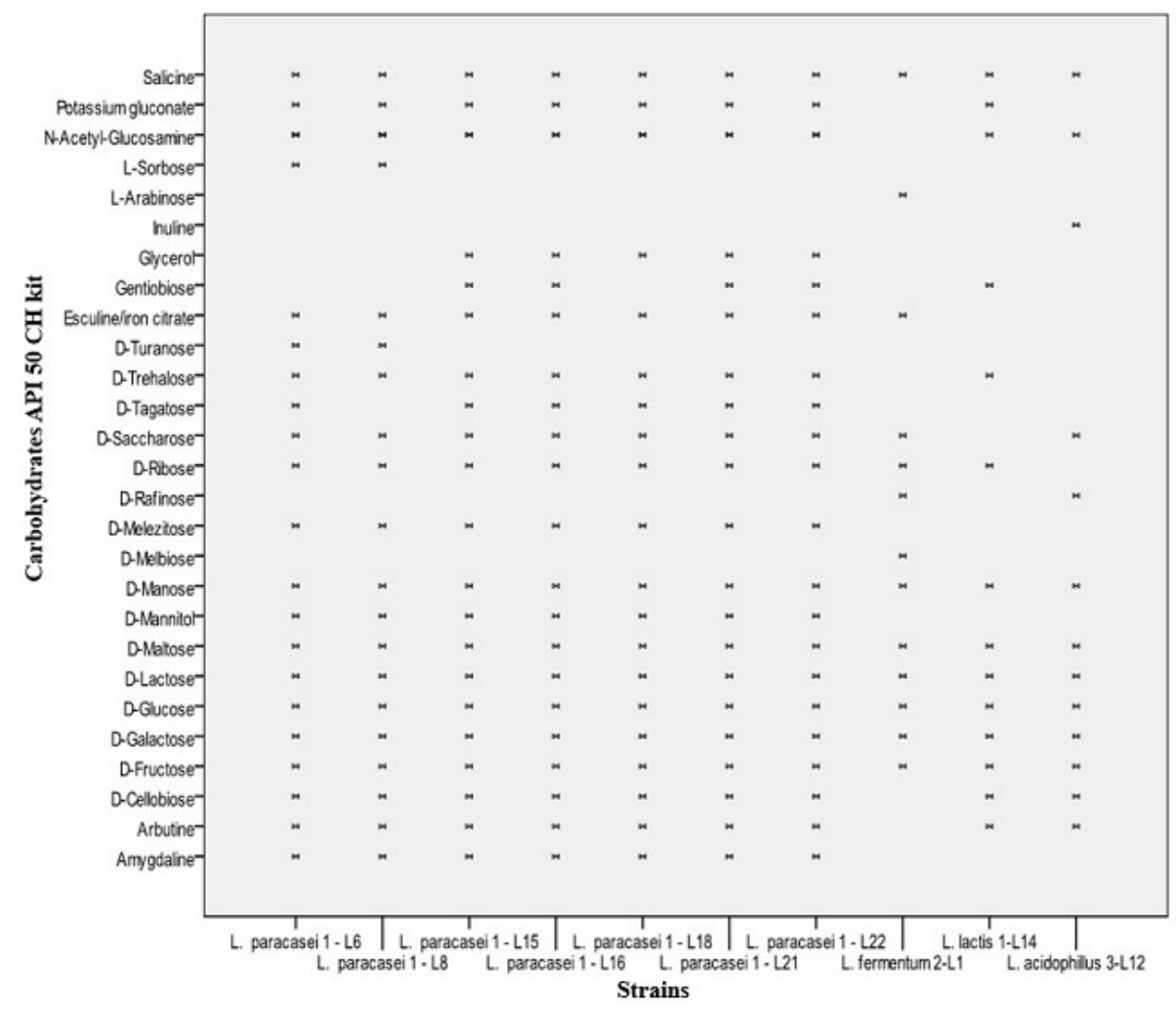

Figure 1. Strain's carbohydrates fermentation patterns on API $50 \mathrm{CH}$ panel

Lactoccocus lactis ssp lactis strain (L14) fermented salicine, n-acetylglucosamine, gluconate, gentiobiose, trehalose, ribose, mannose, galactose, glucose, fructose, lactose, arbutine, cellobiose, maltose. Doutoum et al., (2013) have identified multiple Lactococcus lactis strains on API $20 \mathrm{CH}$ kit with different percentages of confidence ( SLA $1-43.8 \%$, SLA6- $59.9 \%$, SLA3- 95.5\%, SLA4-97.5\%, SLA5-99.8\%). Carbohydrate fermentation pattern for our strain on API $50 \mathrm{CH}$ revealed affinity also for rhamnose, mannitol, sorbitol, esculine, sucrose hence there is also a variability in the biochemical characteristics of Lactoccocus lactis ssp lactis.

Applying hierarchical clustering method (Figure 2) we estimated the similarity among the ten strains carbohydrate fermentation profiles. Pearson's correlations coefficients values on strain pairs were studied regarding these patterns. We have used average linkage between groups with Apriori clustering algorithm to build the dendrogram for Lactobacillus

Vol. XVIII (2014), no. 2 
isolates according to similar approaches described by other authors Dimitonova et al., (2008). Cluster analysis indicated two main components when classifying the isolates: the first cluster included Lactobacillus paracasei strains (L6, L8, L15, L16, L18, L21, L22), the second one comprising Lactobacillus fermentum strain(L1), Lactoccocus lactis ssp lactis strain (L14), Lactobacillus acidophilus strain (L12).

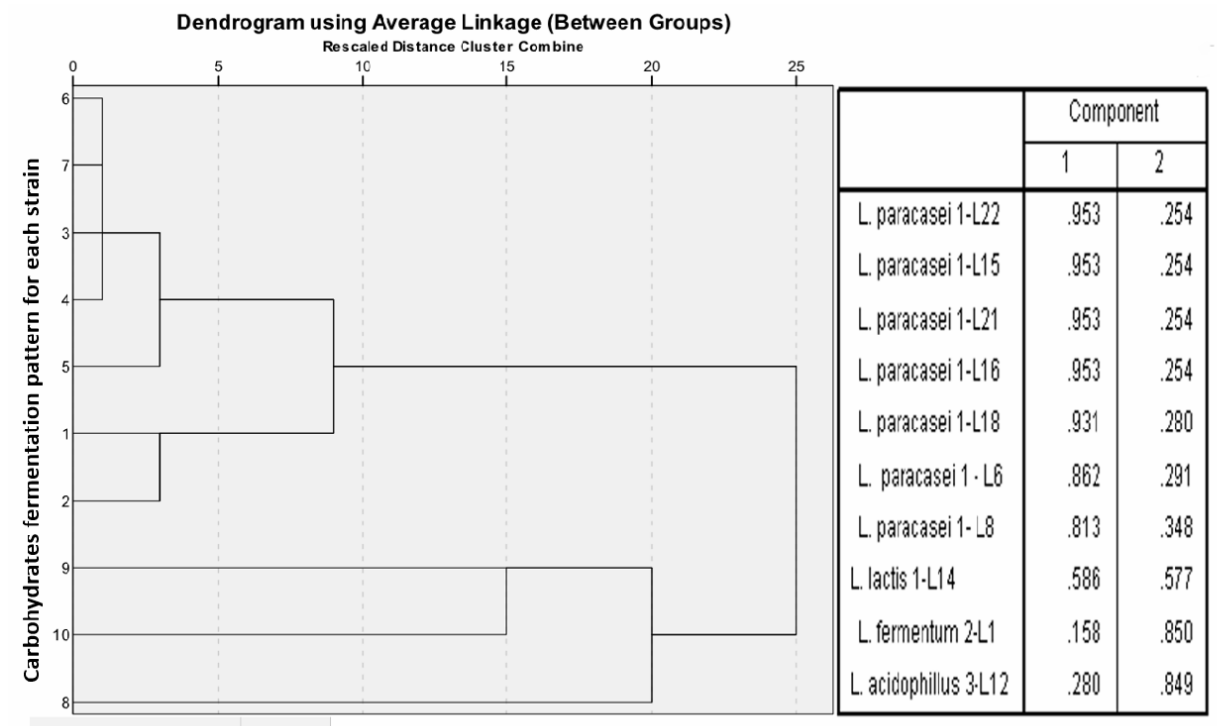

Figure 2. Dendrogram among the ten isolates from human milk (Apriori clustering algorithm)

Despite similar patterns in carbohydrates fermentation and statistically significant Pearson coefficients we could notice some differences between Lactobacillus paracasei ssp paracasei (L6, L8, L15, L16, L18, L21, L22). Glycerol was utilised as substrate for L15, L16,L18,L21,L22, trehalose for all the members, gentiobiose only for L15, L16, sorbose and turanose for L6, L8. There is a considerable metabolic capability for Lactobacillus paracasei species according to Kadere et al., (2012). The affinity for glycerol and trehalose for Lactobacillus paracasei ssp paracasei could explain the survivability to multiple freeze-thaw cycles.

In the other group, besides the common carbohydrates fermentation characteristics, Lactobacillus fermentum biochemical capabilities revealed catabolism of sorbose, esculine, melbiose catabolism whereas Lactobacillus acidophilus expressed affinity for inuline, raffinose and Lactoccocus lactis ssp lactis preferences for trehalose, gentiobiose, gluconate and ribose. Taxonomic identification by API $50 \mathrm{CH}$ kits is still widely used, because it is

Vol. XVIII (2014), no. 2 
practical, easy to perform despite the increasing number of lactic acid bacteria with small differences in biochemical traits (Herbel et al., 2013). Our results overlapped with other studies findings. We have set the confidence interval for identification between $75 \%$ (acceptable) and 100\% (excellent).

Seven probes have been identified as Lactobacillus paracasei ssp paracasei (Figure.3), five strains with a percentage of $99.9 \%$ and a $\mathrm{T}$ index of 0.88 . These strains had a heterofermentative metabolism type. The remaining two isolates were also identified at an excelent rate of $99.8 \%$ and a $\mathrm{T}$ index of 0.94 . In current literature identtification rates for this species are $92.5 \%$ (Karna et al., 2007), 98.15\% (Hyun jue Kim et al., 2006), and $99.9 \%$ (Karna et al., 2007).

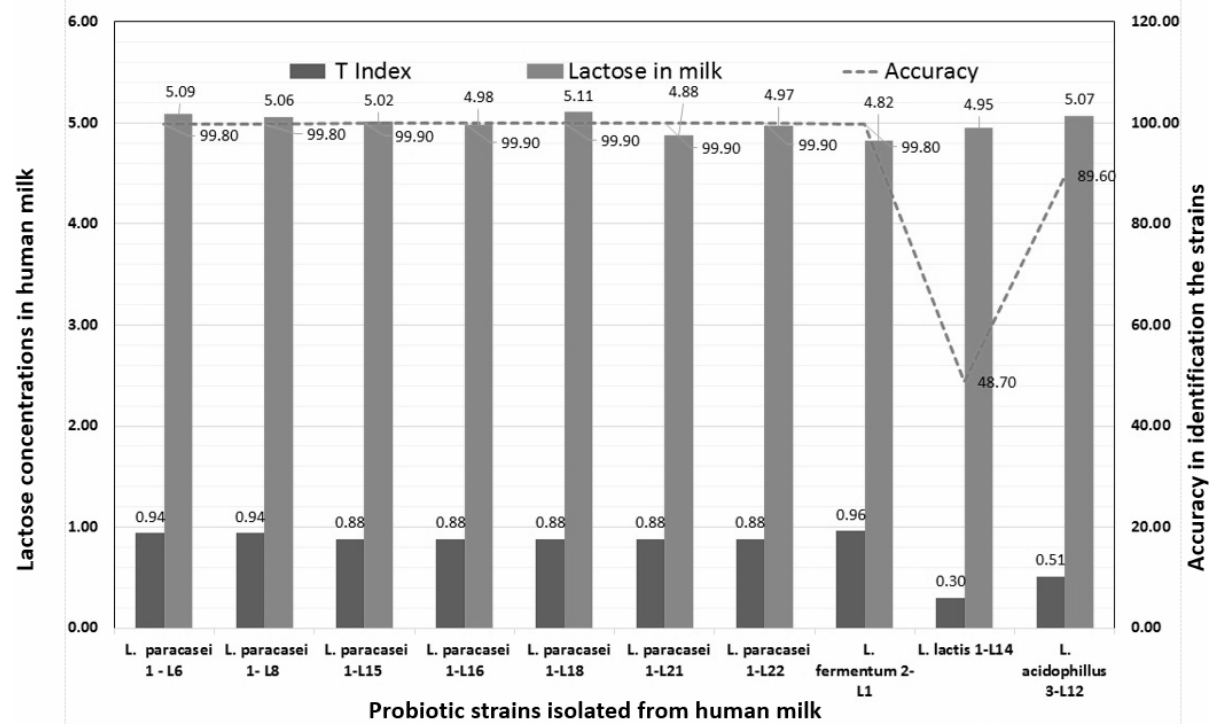

Figure 3. Sinopsis of data among the ten milk probes: lactose concentrations, strain species, strain's identification rate (accuracy), $\mathrm{T}$ index.

Lactobacillus fermentum strain was isolated with a rate of $99.8 \%$ and a T index of 0.96. In a study regarding infant's gut microbiota, Lactobacillus fermentum was isolated from the faeces of infants with an identification rate of $63 \%$ from 55 isolates (Khalil et al., 2007).

Lactobacillus acidophilus strain had a percentage of $89.6 \%$ and a $\mathrm{T}$ index of 0.51, implying a lower taxonomic significance compared with Lactobacillus paracasei ssp paracasei or Lactobacillus fermentum. Sources of isolation and API $50 \mathrm{CH}$ identification of Lactobacillus acidophilus (described in the papers) with good values regarding accuracy (more than 93\%) are represented by : 1. Commercial milk (Karna et al., 2007); 2. Yogurt Acta Universitatis Cibiniensis Series E: FOOD TECHNOLOGY

Vol. XVIII (2014), no. 2 
(Lactobacillus acidophilus LA15, Zheng et al., 2013); 3. Faeces of breast-fed infants (Khalil et al., 2007); 4. Digestive tract of sacrificed chickens, Lactobacillus ssp acidophilus 1 and 3 (Ashraf et al., 2009),(Kizerwetter et al., 2005); 5. Collections of reference for example Lactobacillus acidophilus M 92 (Kos et al., 2008), (Çakmakci et al., 2012). Not all the studies on Lactobacillus acidophilus strain identification rate revealed good percentages. Kizerwetter's findings in species isolated from the digestive tract of sacrificed chickens have shown an unsatisfactory identification percentage of 51\% (Kizerwetter et al., 2005). For this species our identification rate $(89.6 \%)$ is placed between acceptable and good and is close but superior to other results. Lactobacillus acidophilus strains for example, isolated from the faeces of infants were identified with an acceptable value of $84.7 \%$ (Khalil et al., 2007). Lactobacillus acidophilus is described as a reactive or nonreactive strain for all 50 tests in many published papers (Herbel et al., 2013). Lactoccocus lactis ssp lactis identified in our study had a rate below $50 \%$ and was considered unacceptable. There are also reports similar with our results $(43.8 \%)$ (Doutoum et al., 2013) although other authors, Kizerwetter et al(2005) reported admissible values of $84.2 \%$.

Lactobacilli have the posibility of genome expansion or contraction with the deprivation of certain carbohydrate transporters types useful in the process of adapting to the specific environments. It is well established that lactobacilli select one main carbohydrate substrate and lactose from human milk is the most appropriate candidate (Klaenhammer et al.,2007) Regulation of carbohydrate metabolism has been identified in multiple genome studies including Lactobacillus acidophilus NCFM and Lactoccocus lactis (O'Donnell et al.,2013). The authors demonstrated in their review that multiple studies have indicated an increase in the abundance of pyruvate kinase in the presence of lactose. Pyruvate kinase concentrations suggest carbon catabolite repression, promoting the downregulation of genes for nucleotide metabolism. Constant exposure to lactose could influence catabolic genes expression of these lactic acid bacteria by amplifying carbon catabolite repression, possibly causing genome contraction.

We have used Pearson's Correlations applied between lactose levels in human milk, and accuracy in identification or $\mathrm{T}$ index. Pearson's correlations coefficients were neither statistical significant nor showing any tendency in this respect (table 1). Most probably these species use lactose as the most important carbohydrate source in breast milieu (Leloir pathway) but it is likely to possess an important load of pseudogenes (environment specific

Vol. XVIII (2014), no. 2 
genes) in their genomes which could explain phenotipic variability on the fermentation profiles. In our study all of isolated strains have presented affinity for maltose utilisation which according to O'Donnel et al., (2013) is internalised in the bacteria in a phosphorylated form by an enzyme (encoded by a gut activated specific gene).

Table 1. Pearson's Correlations applied between lactose levels in human milk, accuracy in identification and $\mathrm{T}$ index

\begin{tabular}{ccccc}
\multicolumn{5}{c}{ Pearson's Correlations } \\
T index & $\begin{array}{c}\text { Lactose in } \\
\text { milk }\end{array}$ & Accuracy \\
T index & Pearson & 1 & -.033 & $.908^{* *}$ \\
& Correlation & & & \\
& Sig. (2-tailed) & & .929 & .000 \\
Lactose in & N & 10 & 10 & 10 \\
milk & Pearson & -.033 & 1 & .112 \\
& Correlation & & & .757 \\
Accuracy & Sig. (2-tailed) & .929 & 10 & 10 \\
& N & 10 & .112 & 1 \\
& Pearson & $.908^{* *}$ & & \\
& Correlation & & .757 & 10
\end{tabular}

This enzyme works with the maltose phosphotransferase system. We could extrapolate considering that similar mechanisms would explain preferences for a particular carbohydrate substrate for each strain we have isolated. It seems that all of the isolates have shown an important metabolic flexibility being prepared to survive in infant's gut environment.

\section{CONCLUSIONS}

Lactobacilli species from human milk possess considerable adapting capability to survive infant's gut environment. This work has succesfully identified 3 subspecies of Lactobacillus (ssp paracassei 1, ssp acidophilus 3, ssp fermentum 2) with paracassei 1 subspecies as the dominant ones (70\%). Carbohydrate fermentation panel API 50 CHL has shown its reliability in the case of Lactobacillus species identification however due to genetically conditioned strain variances in metabolic performances we suggest that other studies should have an integrated approach (carbohydrates metabolism and functional genomics). Furthermore we believe that API database should be regularly updated based on these studies. Regarding lactose concentrations

Vol. XVIII (2014), no. 2 
influencing the expression of the genes, their regulatory networks and finaly fermentation behaviours on different carbohydrate substrates, we couldn't evidentiate any link between lactose concentrations in the milk probes and carbohydrate metabolic trends. More research data are needed on functional genomics concerning carbon catabolite repression.

\section{ACKNOWLEDGMENTS}

We want to express our gratitude to Pediatric Clinical Hospital from Sibiu for supplying the API $50 \mathrm{CH}$ carbohydrate fermentation strips, API $50 \mathrm{CHL}$ medium, milk probes, lactobacilli isolates used for this study and Lucian Blaga University from Sibiu for supporting this work by a POSDRU 2009, 88/1.5/S/60370 PhD scholarship.

\section{LITERATURE}

1. Agrawal R. (2005) Probiotic: an emerging food supplement with health benefits. Food Biotechnology, 19(3), 227-246; DOI: 10.1080/08905430500316474

2. Ashraf M., Arshad M., Siddique M. and Muhammad G. (2009) In Vitro Screening of Locally Isolated Lactobacillus Species for Probiotic Properties; Pakistan Veterinary Journal., 29(4), 186-190;eISSN: 20747764

3. Boyd M. A. , Antonio May A.D. and Hillier L. Sharon (2005), Comparison of API $50 \mathrm{CH}$ Strips to Whole-Chromosomal DNA Probes for Identification of Lactobacillus Species; Journal of Clinical Microbiology , 43(10), 5309-5311. DOI :10.1128/JCM.43.10.53095311.2005

4. Burgain J., Gaiani C., Linder M., Scher J. (2011) Encapsulation of probiotic living cells : From laboratory scale to industrial applications, Journal of Food Engineering 104(4), 467-483 ; DOI : 10. 1016/j.foodeng.2010.12.031;

5. Çakmakci S., Bülent Ç., Turgut T., Gurses M., Erdogan A. (2012) Probiotic Properties, Sensory Qualities and Storage Stability of Probiotic Banana Yogurts; Turkish Journal Veterinary Animal Science 36(3), 231237, DOI:10.3906/vet-1007-2

6. Chirico G., Marzollo R., Cortinovis S., Fonte C., Gasparoni A., (2008) Antiinfective properties of Human Milk, The Journal of Nutrition: 138(9),1801S-1806S, eISSN :1541-6100;

7. Dimitonova P. S., Bakalov Vladimirov B., Aleksandrova-Georgieva R. N., Danova S. Trifonova (2008), Phenotypic and molecular identification

Vol. XVIII (2014), no. 2 
of lactobacilli isolated from vaginal secretions, Journal of Microbiology, Immunology and Infection, 41(6), 469-477, eISSN: 1995-9133 ;

8. Doutoum A. A., Tidjani A., Abdelaziz Arada I., Tidjani S. M. T, Balde M., Sylla K. S. B., Seydi Mg and Toguebaye B.S.(2013), Identification of the lactic microorganism responsible for milk cogulation in Abeche (Chad), African Journal of Food Science, 7 (5), 103-106, DOI : 10.5897/AJFS12.161;

9. Herbel R. Stefan, Vahjen Wilfried, Wieler Lothar H and Guenther Sebastian; (2013); Timely Approaches to Identify Probiotic Species of the Genus Lactobacillus. Gut Pathogens; 5(1); DOI:10.1186/1757-47495-27

10. Hyun-jue Kim, Han-seung Shin,Woel-kyu Ha, Hee-jin Yang and Soowon Lee (2006), Characterization of Lactic Bacterial Strains Isolated from Raw Milk; Asian-Australasian Journal of Animal Sciences,19(1); 131-136, DOI:10.5713/ajas2011.11177

11. Kadere T.T. , Kutima M.P., (2012), Isolation and Identification of Lactic Acid Bacteria in Coconut Toddy (MNAZI), Journal of Asian Scientific Research , 2(12):807-819, eISSN:2223-1331

12. Karna B.K.L., Emata O.C. and Barraquio V.L. (2007), Lactic Acid and Probiotic Bacteria from Fermented and Probiotic Dairy Products ; Science Diliman 19(2), 23-24, eISSN :2310-9173;

13. Khalil R, El-Halafawy K, Mahrous H, Kamaly K, Frank J, Soda El M (April 2007) Evaluation of the Probiotic Potential of Lactic Acid Bacteria Isolated from Faeces of Breast Fed Infants in Egypt; African Journal of Biotechnology , 6 (7), 939-949, DOI: 10.5897/AJB2007.000-2114;

14. Kizerwetter-Swida M. and Binek M. (2005) Selection of Potentially Probiotic Lactobacillus Strains Towards their Inhibitory Activity against Poultry Enteropathogenic Bacteria Polish Journal of Microbiology, 54 (4), 287-294; eISSN 1733-1331

15. Klaenhammer T. R., Azcarate-Peril M.A., Altermann E., and Barrangou R. (2007) Influence of the Dairy Environment on Gene Expression and Substrate Utilization in Lactic Acid Bacteria, The Journal of Nutrition, 137(3): 748S-750S, eISSN :1541-6100

16. Kos B., Šušković J., Beganović J., Gjuračić K., Frece J., Iannaccone C., Canganella F. (2008). Characterization of the Three Selected Probiotic Strains for the Application in Food Industry, World Journal of Microbiology and Biotechnology 24(5):699-707, DOI 10.1007/s11274007-9528-y;

17. Leuschner R., Kneife W., Vernoux J.-P. , Stanton C. and Aldamiz P. (2002). Methods for the Official Control of Probiotics used as Feed

Vol. XVIII (2014), no. 2 
Additives, volume 2, Luxembourg: European Comission Community Research,;

18. Malek Abd R., Sallehhuddin Bin Hamdan, Hesham A El Enshasy, Nor Zalina Othman, Noor Azwani Zainol, Mohamad R Sarmidi, A Aziz (2010); Production of Lactobacillus salivarius, a new probiotic strain isolated from human breast milk, in semi-industrial scale and studies on its functional characterization; In A. Mendez-Villas(Ed), In Current Research, Technology and Education Topics, Applied Microbiology and Microbial Biotechnology (pp.1196-1204 ) Spain: Formatex Research Center

19. Marroki A., Zúñiga M., Kihal M., Pérez-Martínez G. (2011), Characterization of Lactobacillus from Algerian goat's milk base don phenotypic, 16S rDNA sequencing and their technological properties, Brazilian Journal of Microbiology, 42(1), 158-171, DOI:101590/S1517838222011000100020

20. O’Donnell M. M, O'Toole P. W and Ross R. P. (2013), Catabolic flexibility of mammalian-associated lactobacilli, Microbial Cell Factories,12(48), DOI:10.1186/1475-2859-12-48;

21. Yavuzdurmaz H., Sebnem H. (2011) Selection of potential probiotic lactobacillus strains from human milk; In Food Product Engineering \&Functional Foods: 11th International Congress on Engineering and Food, (pp2043-2044), Athens, Greece: Cosmoware

22. Zavisic G, Petricevic S, Radulovic Z, Begovic J, Golic N, Topisirovic L, Strahinic I. (2012) ;Probiotic Features of Two Oral Lactobacillus Isolates, Brazilian Journal of Microbiology, 43(1), 418-428; DOI:10.1590/S1517-83822012000100050

23. ZhengY., LuY., Wang J., Yang L., Pan C., Huang Y. (2013), Probiotic Properties of Lactobacillus Strains Isolated from Tibetan Kefir Grains; PLOS ONE 8(7); DOI : 10.371/journal.pone.0069868

Vol. XVIII (2014), no. 2 